\title{
СИСТЕМНЫЙ АНАЛИЗ И ГИПОТЕЗА ФИЗИЧЕСКОЙ КАРТИНЫ ПРОЦЕССА ОЧИСТКИ ПОГРУЖНОГО ОБОРУДОВАНИЯ СКВАЖИН С ПРИМЕНЕНИЕМ МОДЕРНИЗИРОВАННОГО ОБРАТНОГО КЛАПАНА
}

\author{
Азеев Александр Александрович', \\ 3-a@li.ru \\ Булчаев Нурди Джамалайлович', \\ nbulchaev@yandex.ru
}

\author{
Безверхая Елена Владимировна', \\ bezverhaya.elena@mail.ru
' Институт нефти и газа Сибирского Федерального университета, Россия, 660041, г. Красноярск, пр. Свободный, 79.

\begin{abstract}
Актуальность. Анализ технических средств и методов борьбы с пескопроявлениями дает основание предполагать наличие значительных резервов повышения их эффективности. В частности, установлено, что большинство методов снижения влияния механических примесей на работу насосного оборудования в настоящее время либо не решают проблему кардинально, либо не носят долговременного характера. Гидродинамическая очистка погружного оборудования, несмотря на свою эффективность, невозможна без проведения спускоподъемных операций. Альтернативой этого способа являются устройства клапанного типа. Однако их применение сдерживается из-за того, что не решен вопрос подбора оптимальных конструктивных параметров данных устройств. В связи с вышеизложенным есть необходимость проведения соответствующих теоретических исследований. Цель: провести системный анализ и предложить гипотезу физической картины процесса очистки погружного оборудования скважин с применением модернизированного обратного клапана.

Методы: системный анализ процесса очистки погружного оборудования скважин.

Результаты. Системный анализ работы очистных установок клапанного типа показывает, что из всех элементов внутренней структуры наибольшее число связей имеют параметры специального очистного оборудования. Следовательно, дальнейшее изучение рассматриваемой системы связано с выделением соответствующей подсистемы, определяющей только процесс очистки, а также с выяснением физической картины этого процесса. Предложена гипотеза физической картины процесса очистки, учитывая которую, можно заметить, что в подсистеме «Условия эксплуатации - конструктивные и технологические параметры клапана - производительность» в большей степени реализуется основное назначение всей системы. Выделение этой подсистемы отдельно позволило в общем виде найти взаимосвязь производительности процесса очистки и основных элементов внутренней структуры этого процесса. Практическая польза найденной взаимосвязи заключается в возможности обоснованного поиска оптимальных конструктивных параметров установки клапанного типа и скорости очистного потока через нее, в зависимости от основных факторов.
\end{abstract}

Ключевые слова:

Обратный клапан, добывающая скважина, очистка, фильтр, центробежный насос.

\section{Введение}

Анализируя существующие способы промывки погружного оборудования [1-15], включая область забоя скважин, можно отметить следующее. Вопервых, все известные методы очистки в настоящее время либо не решают проблему вредного влияния механических примесей, солей и отложений кардинально, либо не носят долговременного характера. В частности, что касается новых перспективных механических методов борьбы с пескопроявлениями: несмотря на столь обнадеживающие результаты первых опытов использования сепараторов песка [8], нельзя говорить о целесообразности их массового использования для защиты установки электроцентробежного насоса (ЭЦН) и штангового глубинного насоса во всех без исключения скважинах с терригенными коллекторами. Перспективно их применение после гидравлического разрыва пласта, а также в одной компоновке с ЭЦН на сильно обводненных скважинах, находящихся на грани рентабельности, так как это позво- лит использовать более экономичный вариант исполнения насоса. Кроме того, для всех методов очистки [1, 5-10] характерно использование трудоемких и длительных спускоподъемных операций.

Во-вторых, наиболее эффективным из всех существующих способов очистки погружного оборудования скважин является гидродинамический способ очистки специальными насадками высокого давления [10-15]. Однако их применение невозможно без проведения спускоподъемных операций. В-третьих, единственное решение, которое позволяет сократить необходимость частого выполнения спускоподъемных операций связано с применением устройств клапанного типа, являющихся, в частности, модернизацией стандартного трехпозиционного обратного клапана [16-18]. Сoгласно работам $[16,17]$, обратное открывание модернизированного обратного клапана происходит без длительного повышения давления в насоснокомпрессорной трубе (НКТ) за счет опускания на 


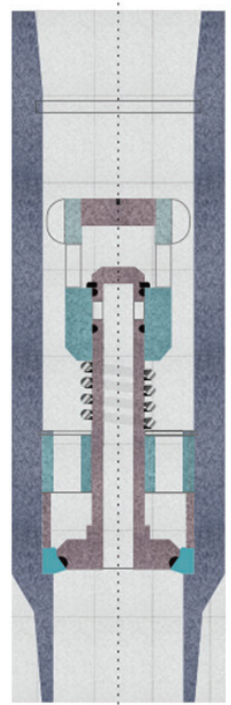

$a / a$

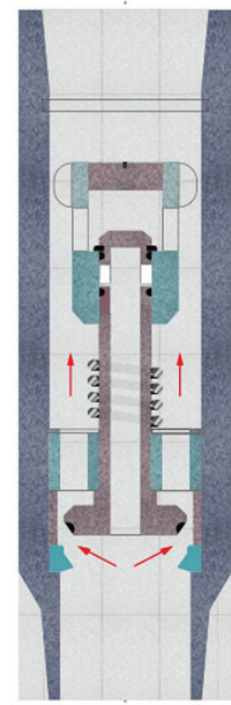

$\sigma / b$

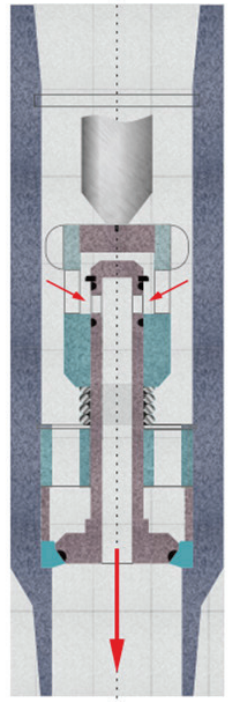

$B / c$

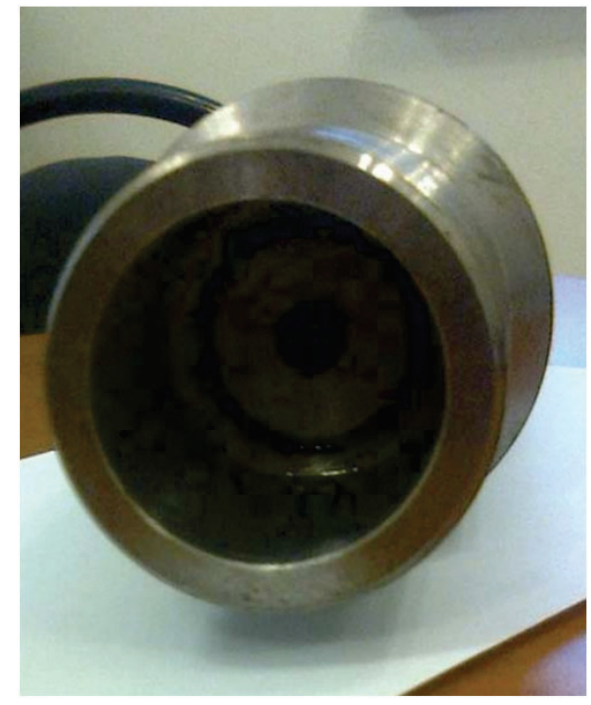

$2 / d$

Рис. 1. Модернизированный обратный клапан: а) остановка установки электроцентробежного насоса (УЭЦН ); б) работа УЭЦН; в) спуск груза для промывки приемной сетки УЭЦН; г) фотография клапана

Fig. 1. Improved backpressure valve: a) stop of Electrical Submersible Pumping (ESP System ); b) ESP System operation; c) descent of freight for washing of a reception grid of the ESP System; d) valve photo

скребковой проволоке груза через лубрикатор (рис. 1). Этот груз, благодаря своему весу, превышающему силу упругости пружины, сожмет ее и переместит втулку вниз до положения, соответствующего обратному открыванию клапана. По истечении определенного времени груз приподнимают, и втулка под действием сжатой пружины возвращается в крайнее верхнее положение, при котором прекращается переток жидкости из НКТ в скважину.

Анализ результатов предыдущих исследований $[8,10,11,16,19-23]$ дает основание предполагать наличие значительных резервов повышения эффективности технических средств и методов борьбы с пескопроявлениями. В работах $[11,16]$ не решен вопрос подбора конструктивных параметров устройств клапанного типа, при которых обеспечиваются минимальные гидравлические сопротивления. Для поиска решения этого вопроса есть необходимость проведения соответствующих теоретических исследований.

\section{Системный анализ процесса очистки}

Структуру процесса очистки погружного оборудования скважин, с позиции системного подхода, целесообразно рассмотреть в виде подсистем верхнего, среднего и нижнего уровня, имеющих между собой соответствующие функциональные связи. Пример подобной структуры, приведенный на рис. 2, позволяет наглядно показать взаимосвязь конструктивных, технологических и технико-экономических показателей очистных установок.

Из рис. 2 видно, что из всех элементов внутренней структуры наибольшее число связей имеют па- раметры специального рабочего оборудования. Следовательно, для их дальнейшего изучения необходимо выделить соответствующую подсистему, определяющую только процесс очистки, а также выяснить физическую картину этого процесса. На основании результатов предварительных исследований [16], в качестве допущения, в упрощенном виде физическую картину процесса очистки можно представить как совокупность отдельных физических процессов, чередующихся в пространстве друг за другом (рис. 3).

Технология очистки приемной сетки насоса происходит следующим образом. С помощью груза, подаваемого внутрь насосно-компрессорной трубы через лубрикатор на скребковой проволоке, производится сжатие пружины исполнительной втулки модернизированного обратного клапана, что вызывает свободный переток жидкости с устья к приемному фильтру насоса. Далее последовательность происходящих друг за другом физических процессов включает в себя следующие этапы.

На первом этапе поток очистной жидкости, подаваемый с устья насосной установкой, проходит по насосно-компрессорной трубе и встречает на своем пути клапанный механизм, пройдя через который на выходе образует уже несколько потоков более высокого давления. На втором этапе полученные потоки жидкости смешиваются и перемещаются по насосным трубам далее, проходя через погружной центробежный насос до его приемных фильтров. При условии постоянства величины гидравлических сопротивлений в элементах клапанного механизма и погружного насоса общие потери на трение в ходе продвижения очистного потока 


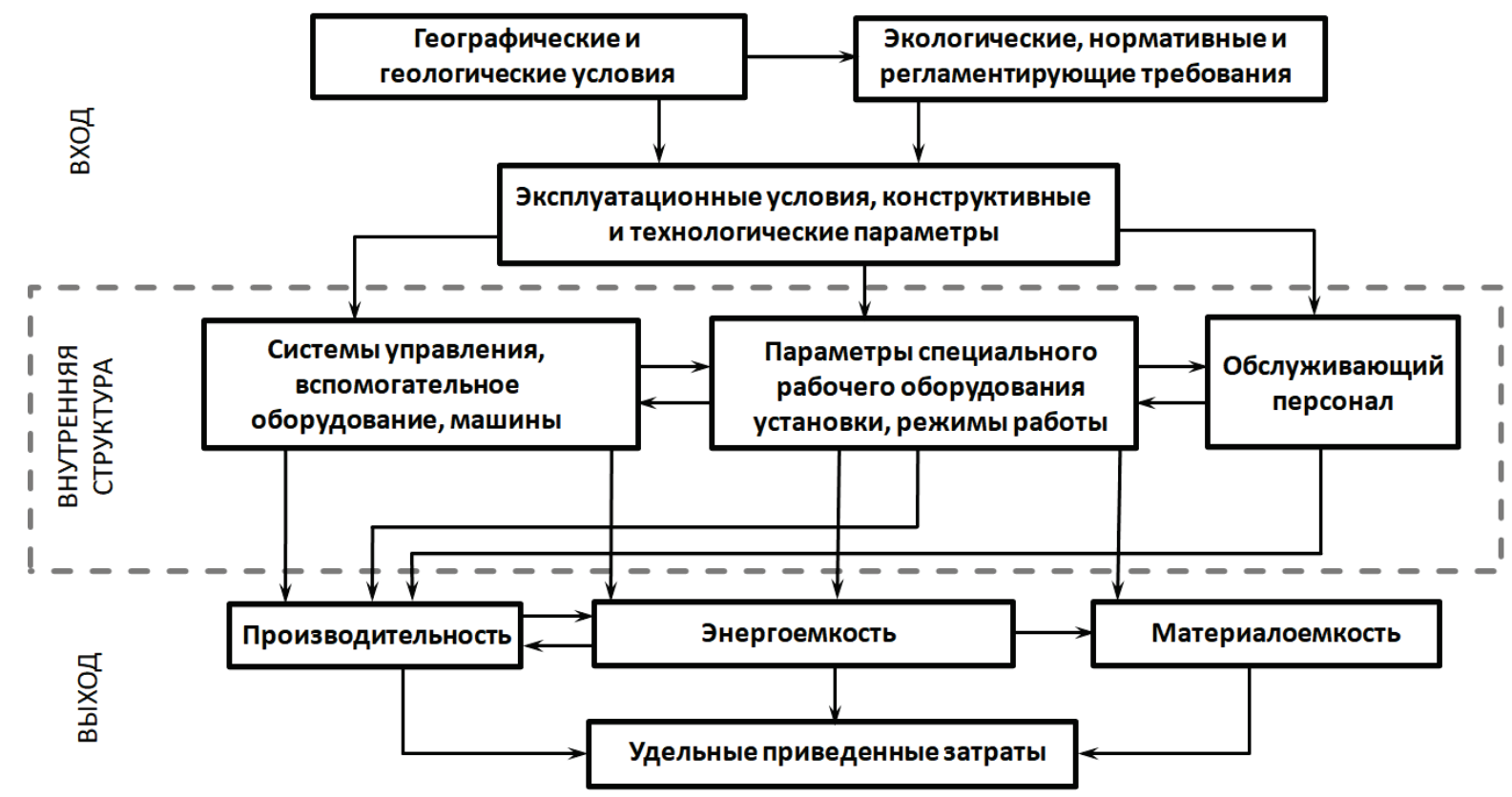

Pис. 2. Структурная схема процесса работы гидродинамической установки клапанного типа в ходе очистки погружного оборудования скважины

Fig. 2. Block diagram of the valve type hydrodynamic device operation at purification of well submersible equipment

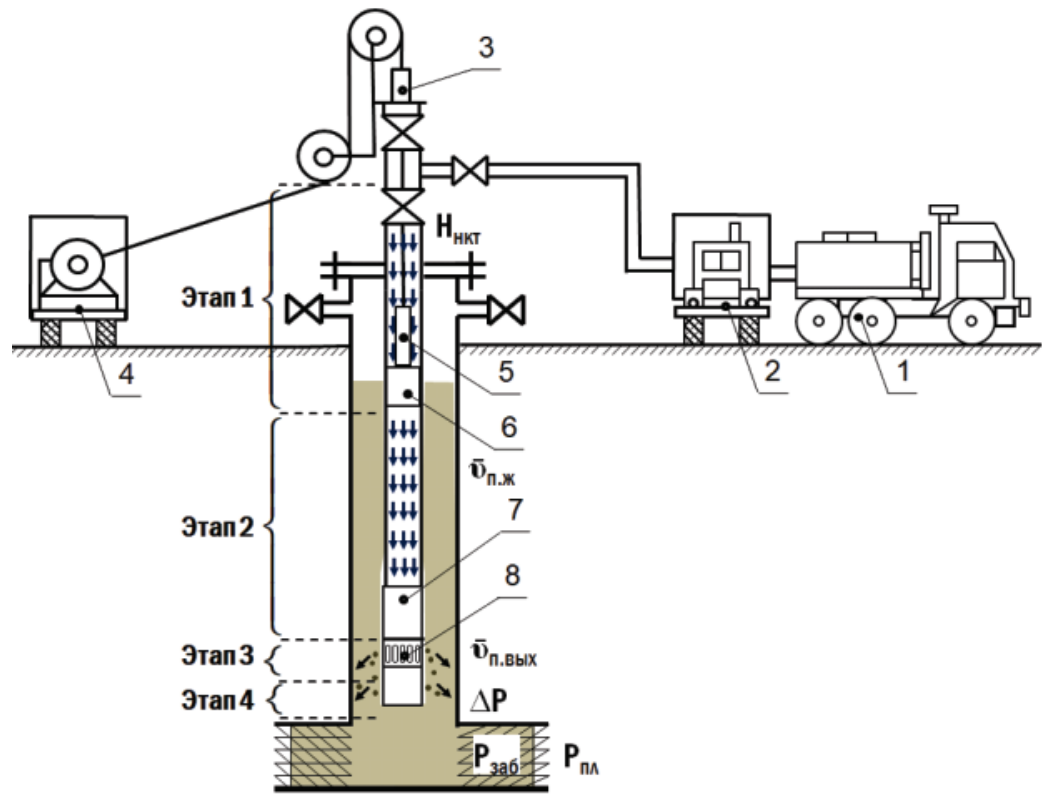

Рис. 3. Схема к расчету процесса очистки погружного оборудования скважины с применением модернизированного обратного клапана с функиией двухстороннего перепуска жидкости через себя: 1 - автоцистерна; 2 - насосный агрегат; 3 - лубрикатор; 4 - лебедка; 5 - груз; 6 - модернизированный клапан; 7 - центробежный насос; 8 - приемный фильтр

Fig. 3. Scheme to calculation of well submersible equipment purification using the upgraded backpressure valve with the function of bilateral restart-up of liquid: 1 is the tanker truck; 2 is the pump unit; 3 is the lubricator; 4 is the winch; 5 is the freight; 6 is the upgraded valve; 7 is the centrifugal pump; 8 is the reception filter

будут расти в прямой пропорции к длине пройденного пути. В связи с этим на некотором расстоянии от устьевой насосной установки давление потока на отложение внутри приемного фильтра может стать равным связующим усилиям между частицами отложения, следовательно, его разрушение бу- дет затруднено. При этом в зависимости от степени загрязнения фильтра и связующих сил между частицами отложения поток начнет перемещаться либо через фильтр в межтрубное пространство, либо в обратную сторону, вызывая повышение уровня жидкости в насосно-компрессорной трубе перед 


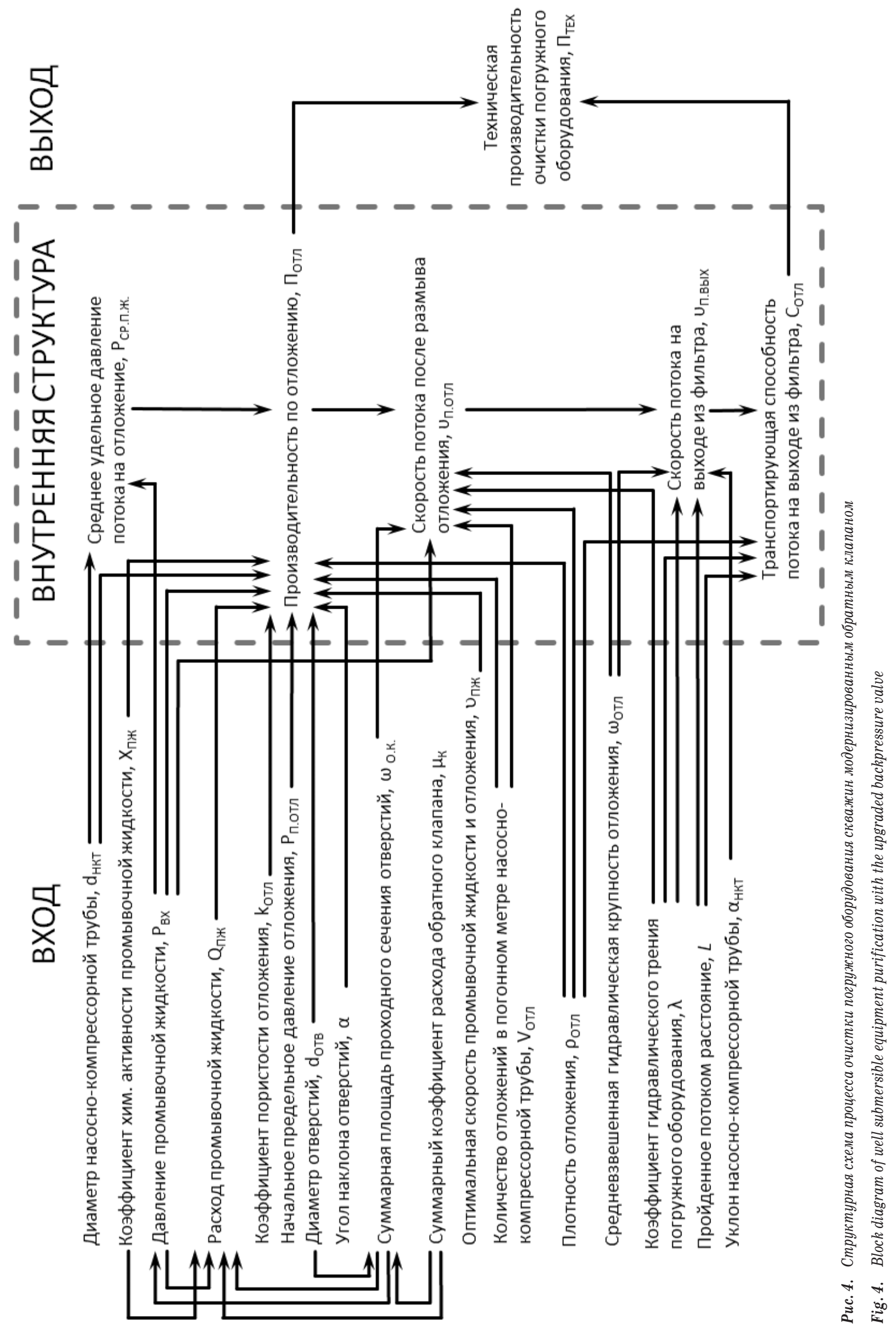


преградой. Возможен также комбинированный вариант, с частичным истечением очистного потока через фильтр и изменением гидростатического уровня. На третьем этапе очистная жидкость, взаимодействуя с отложениями приемной сетки фильтра, начинает их разрушать, образуя многофазный поток, включающий частицы отложения, очистного состава и пластового флюида. На четвертом этапе многофазный поток по межтрубному пространству перемещается ниже с замедлением скорости, при этом твердые частицы выпадают в виде осадка в зумпф.

Учитывая описанную выше гипотезу физической картины рабочего процесса, можно заметить, что в подсистеме «Условия эксплуатации, конструктивные и технологические параметры - параметры специального рабочего оборудования производительность» в большей степени реализуется основное назначение системы. Эту подсистему целесообразно выделить отдельно, например, согласно рис. 4.

Элементы внутренней структуры подсистемы описывают параметры процессов, связанных с работой специального рабочего оборудования, к которому относится клапанный механизм. В частности, к элементам внутренней структуры относятся: среднее удельное давление потока на отложение $\left(P_{\text {ср.п.ж}}\right)$, производительность по отложению $\left(\Pi_{\text {отл }}\right)$, скорость потока после размыва отложения $\left(v_{\text {п.от. }}\right)$, скорость потока на выходе из фильтра $\left(v_{\text {п. }}\right.$ вых и т. д. Параметры входа имеют прямые свяи с параметрами внутренней структуры и через них влияют на выход подсистемы, т. е. на техническую производительность очистного оборудования.

Формулу технической производительности [24] установки клапанного типа для очистки погружного оборудования в общем виде можно записать следующим образом:

$$
\Pi_{\text {тех }}=f\left(\begin{array}{l}
d_{\text {нкт }}, X_{\text {пж }}, P_{\text {вх }}, k_{\text {отл }}, P_{\text {п.отл }}, d_{\text {отв }}, \\
\alpha, \omega_{\text {о.к }}, \mu_{\mathrm{K}}, V_{\text {отл }}, v_{\text {п.ж }}, \rho_{\text {отл }}, \lambda, L
\end{array}\right),
$$

где $d_{\text {нкт }}-$ внутренний диаметр НКТ, м; $X_{\text {пж }}$ - коэффициент химической активности промывочной жидкости; $P_{\text {вх }}$ - давление на входе в обратный клапан, МПа; $Q_{\text {п.ж }}$ - расход промывочной жидкости через одно из отверстий, $\mathrm{m}^{3} /$ ч $k_{\text {отл }}$ - коэффициент пористости отложения; $P_{\text {полл }}-$ минимальное предельное давление разрушения отложений, $\mathrm{H} ; d_{\text {отв }}-$ диаметр отверстий в корпусе обратного клапана, м; $\alpha$ - угол наклона отверстий к оси клапана и НКТ, град; $\omega_{\text {о.к }}$ - площадь поперечного сечения отверстия обратного клапана, м $^{2} ; \mu_{\mathrm{\kappa}}$ - суммарный коэффициент расхода обратного клапана; $V_{\text {огл }}$ - объем отложений в погонном метре НКТ или фильтров, $\mathrm{M}^{3} ; v_{\text {п.ж }}$ - оптимальная скорость движения смеси очистной жидкости и отложения, с выпадением осадка за пределами очищаемой зоны, м/ч; $\rho_{\text {огл }}$ плотность отложения, кг $/ \mathrm{m}^{3} ; \lambda$ - коэффициент гидравлического трения погружного оборудования; $L$ - пройденное потоком расстояние, м.
С целью уточнения выражения (1) примем, что достижение основной цели выделенной подсистемы на рис. 4 обеспечивается при соблюдении следующих ограничений и условий:

- отложение полностью удаляется с очищаемого погружного оборудования во всем диапазоне изменения скорости очистного потока, т. е. производительность по удалению отложения равна $\Pi_{\text {от. }}=v_{\text {пा.ж }} V_{\text {от.л }}$;

- толщина и свойства отложения одинаковы на всех внутренних поверхностях очищаемого оборудования и области фильтра на приеме наcoca: $k_{\text {олл }}, P_{\text {п.олл }}, V_{\text {огл }}, \rho_{\text {огл }}, \omega_{\text {огл }}=$ const;

- гидравлические и геометрические характеристики погружного оборудования постоянны на всем протяжении в скважине: $d_{\text {нкт }}, \lambda=$ const;

- параметры специального клапана являются постоянными: $d_{\text {отв }}, \alpha, \omega_{0 . \mathrm{k}}, \mu_{\mathrm{k}}=$ const;

- давление, расход и очистные свойства потока газожидкостной смеси, обеспечиваемые комплексом оборудования, постоянны и соответствуют необходимым и достаточным затратам энергии для удаления отложения: $P_{\text {вх }}, Q_{\text {п.ж }}$, $X_{\text {пж }}=$ const;

- влиянием на процесс очистки забойного и пластового давления пренебрегается.

Таким образом, если параметры клапанного устройства $d_{\text {отв }}, \alpha, \omega_{\text {о.к }}, \mu_{\mathrm{k}}$ неизменны в ходе всего процесса очистки подземного оборудования, имеющего постоянные параметры $d_{\text {нкт }}, \lambda, k_{\text {олл }}, P_{\text {п.отл }}$, $V_{\text {огл }}, \rho_{\text {огл }}, \omega_{\text {огл }}$, что обеспечивает постоянство параметров $P_{\text {вх }}, Q_{\text {п.ж }}$, при постоянной $X_{\text {пж }}$, то скорость очистной смеси можно представить как функцию расстояния $L$, пройденного потоком жидкости через подземное оборудование [24]:

$$
v_{\text {п.ж }}=f(L) \text {. }
$$

Отсюда следует, что общая техническая производительность [24] очистной установки клапанного типа и производительность по удалению отложения при неизменных значениях входных параметров будут равны между собой и функционально зависеть от пройденного потоком расстояния:

$$
\left\{\begin{array}{l}
\Pi_{\text {тех }}=\Pi_{\text {отл }}=v_{\text {п.ж }} V_{\text {отл }} \\
\Pi_{\text {тех }}=f(L) .
\end{array}\right.
$$

Практический интерес поиска оптимальной скорости очистного потока $v_{\text {п.ж }}$ через установку клапанного типа в зависимости от основных факторов процесса заключается в построении номограмм, пользуясь которыми можно выбирать скорость этого потока для скважины конкретной глубины, диаметра и степени зарастания отложениями.

Технология очистки с применением модернизированного обратного клапана, изготовленного с учетом описанных выше формул, была испытана на одной из скважин НГДУ «Туймазанефть». Дебит скважины после 146 сут эксплуатации снизился с 47 до 29 м $^{3}$ /сут. Расчетный вес груза для срабатывания клапана составил 15,5 кг. После оста- 
новки скважины и обратной циркуляции жидкости из НКТ в скважину в течение 10 мин ее вновь запустили. Новый дебит составил $42 \mathrm{~m}^{3} /$ сут, т. е. восстановился на $89 \%$.

\section{Заключение}

Системный подход к процессу очистки погружного оборудования скважин гидродинамическим

\section{СПИСОК ЛИТЕРАТУРЫ}

1. Апасов Р.Т., Шошаева З.А. Способы защиты от механических примесей в добываемой жидкости / Инновации в науке: научный журнал. - 2017. - № 1 (62). - С. 86-88.

2. Кадетов А.В. Технологии НПФ «Пакер» для осложненного фонда скважин // Инженерная практика. - 2018. - № 4. C. $73-75$.

3. Валиханов А.В. Вопросы подъема обводненной и безводной нефти фонтанным и насосным способами. - Казань: Таткнигоиздат, 1971. - $148 \mathrm{c.}$

4. Мищенко И.Т. Скважинная добыча нефти. - М.: Нефть и газ, 2003. $-816 \mathrm{c}$.

5. Михайлов А.Г. Комплексная защита скважинного оборудования при пескопроявлении в 000 «РН-Пурнефтегаз» // Территория нефтегаз. - 2010. - № 12. - С. 84-89.

6. Ашрафьян М.О. Совершенствование конструкций забоев скважин. - М.: Недра, 1986. - 156 с.

7. Магадова Л.А. Разработка композиции для крепления призабойной зоны пласта в скважинах подземных хранилищ газа // Территория НЕФТЕГАЗ» . - 2012. - № 5. - С. 63-67.

8. Якимов С.Б. Сепараторы песка для защиты погружных насосов. Текущая ситуация и перспективы применения технологии // Территория НЕФТЕГАЗ. - 2014. - № 2. - С. 44-58.

9. Атрушкевич С.А. Эжекторная очистка скважин. Опыт разработки и применения // Время колтюбинга. Время ГРП. 2017. - № 3 (061). - C. $26-27$.

10. ACTive- Семейство активных сервисов ГНКТ // Schlumberger. 2019. URL: https://www.slb.ru/upload/iblock/4b5/active.pdf (дата обращения 28.05.2018).

11. Bohnhorst D. Hydrodynamische Kanalreinigungsgerate // Stadtehygiene. - 1961. - № 11. - S. 233-240.

12. Integrated Wellbore Cleanout Systems: Improving Efficiency and Reducing Risk / A. Ali, C.G. Blount, S. Hill, J. Pokhriyal, X. Weng, M.J. Loveland, S. Mokhtar, J. Pedota, M. Rodsjo, R. Rolovic, W. Zhou // Oilfield Review. - 2005. - V. 17. - № 2. P. 4-13.

13. Pipe cleaning nozzle: Pat. 3744723 US on the invention, Int. Cl. B05b1/26, 1973.

\section{Информация об авторах}

Азеев A.A., кандидат технических наук, доцент кафедры разработки и эксплуатации нефтяных и газовых месторождений Института нефти и газа Сибирского Федерального университета.

Булчаев Н.Д., кандидат технических наук, заведующий кафедрой разработки и эксплуатации нефтяных и газовых месторождений Института нефти и газа Сибирского Федерального университета.

Безверхая E.B., кандидат технических наук, доцент кафедры разработки и эксплуатации нефтяных и газовых месторождений Института нефти и газа Сибирского Федерального университета. способом позволил выявить основные элементы процесса и их взаимосвязь. Показана практическая польза поиска оптимальных конструктивных параметров установки клапанного типа и скорости очистного потока через нее, в зависимости от глубины скважины, диаметра насосно-компрессорной трубы и степени зарастания отложениями приемной сетки фильтра электроцентробежного насоса.

14. Nozzle: Pat. 3814330 US on the invention, Int. Cl. B05b1/14, 1974.

15. Vorrichtung zum Reinigen von Rohren, Kanalen, Behaltern sowie der Siebe und Filze von Papiermaschinen: Pat. $400028 \mathrm{CH}$ hauptpatent, Int. Cl. E03F, 1966.

16. Булчаев Н.Д. Защита насосного оборудования нефтяных скважин в осложненных условиях эксплуатации: монография. Красноярск: Сиб. федер. ун-т, 2015. - 138 с.

17. Клапан обратный электроцентробежной установки и способ очистки фильтра на приеме насоса: пат. № 2544930 Рос. Федерация. № 2013142546/03; заявл. 17.09.13; опубл. 20.03.2015, Бюл. № 8. -9 c.

18. Азеев А.А. Автоматизация процесса очистки внутрискважинного оборудования на основе клапана с аналоговым механизмом // Газовая промышленность. - 2016. - № 4. - С. 82-87.

19. Penberthy W.L., Shaughnessy C.M. Sand Control. S P E Series on Special Topics, V. 1. Society of Petroleum Engineers Publ., 1992. $-98 \mathrm{p}$.

20. Practical Approaches to Sand Management / A. Acock, T. O'rourke, D. Shirmboh et al. // Oilfield Review. - 2004. - V. 16. № 1. - P. 10-27.

21. Harrisberger C. Jr, Harrisberger W. Stability of sand arches: a key to sand control // Journal of Petroleum Technology. 1970. - V. 22 (7). - P. 821-829.

22. Смольников С.В. Повышение эффективности эксплуатации продуктивных пластов, сложенных слабосцементированными песчаниками: автореф. дис. ... канд. техн. наук. - М., 2015. $24 \mathrm{c}$.

23. Литвиненко К.В. Прогнозирование технического состояния УЭЦН в условиях интенсивного выноса мехпримесей: автореф. дис. ... канд. техн. наук. - Уфа, 2016. - 24 с.

24. Азеев А.А. Повышение производительности комплекса оборудования для бестраншейного ремонта трубопроводов способом комбинированного торообразного рукава: автореф. дис. ... канд. техн. наук. - Братск, 2011. - 23 с.

Поступила 29.05.2018 г.

месторожденй Институга нефти и газа Сибирского Федерального университета. 


\title{
SYSTEMATIC ANALYSIS AND HYPOTHESIS OF PHYSICAL PATTERN OF WELL SUBMERSIBLE EQUIPMENT PURIFICATION USING THE UPGRADED BACKPRESSURE VALVE
}

\author{
Alexander A. Azeev', \\ 3-a@li.ru \\ Nurdi J. Bulchaev', \\ nbulchaev@yandex.ru \\ Elena V. Bezverkhaya', \\ bezverhaya.elena@mail.ru \\ ' Siberian Federal University,
82, Svobodny avenue, Krasnoyarsk, 660041, Russia.
}

Relevance. Analysis of technical means and methods of controlling sand entry allows supposing the presence of significant reserves to improve their effectiveness. In particular, it was found that most methods of reducing the influence of mechanical impurities on performance of pumping equipment currently or do not solve the problem fundamentally, or do not have a long-term nature. Hydrodynamic cleaning of submersible equipment, despite its efficiency, is impossible without tripping. The alternative to this method is the device of the valve type. However, their application is constrained because of the unsolved issue of selection of optimal design parameters of these devices. In this relation, there is a need for appropriate theoretical research.

The main aim of the research is to carry out a system analysis and to propose a hypothesis of physical pattern of well submersible equipment purification using the upgraded backpressure valve.

Methods: systematic analysis of well submersible equipment purification.

Results. System analysis of operation of valve-type water treatment plants shows that the parameters of a special purification equipment have the largest number of relations among all the elements of the internal structure. Therefore, further study of the system is related to selection of corresponding subsystem, which determines only purification as well as to determination of this process physical pattern. The authors have proposed the hypothesis of purification physical nature. Taking into account this pattern, you can notice that the main aim of the entire system is implemented in the subsystem "Operating conditions - constructive and technological parameters of valve performance». Identification of this subsystem allowed in general determining the relationship between purification performance and the main elements of the internal structure of this process. Practical use of the found interrelations is in possibility of informed search for optimal design parameters of the valve type device and purification flow rate through it, depending on the main factors.

Key words:

Backpressure valve, producing well, purification, filter, electrical submersible pumping.

\section{REFERENCES}

1. Apasov R.T., Shoshaeva Z.A. Ways to protect against mechanical impurities in the produced fluid. Innovatsii $v$ nauke: nauchny zhurnal, 2017, no. 1 (62), pp. 86-88. In Rus.

2. Kadetov A.V. Technologies NPF «Packer» for a complicated well stock. Inzhenernaya praktika, 2018, no. 4, pp. 73-75. In Rus.

3. Valikhanov A.V. Voprosy podema obvodnennoy i bezvodnoy nefti fontannym i nasosnym sposobami [The issues of lifting watered and anhydrous oil in a fountain and pumping way]. Kazan, Tatknigoizdat Publ., 1971. $148 \mathrm{p}$.

4. Mishchenko I.T. Skvazhinnaya dobycha nefti [Downhole oil production]. Moscow, Neft i gaz Publ., 2003. 816 p.

5. Mikhaylov A.G. Complex protection of the borehole equipment at sand entry in Ltd company «RN-Purneftegaz. Territoriya NEFTEGAZ, 2010, no. 12, pp. 84-89. In Rus.

6. Ashrafyan M.O. Sovershenstvovanie konstruktsy zaboev skvazhin [Perfection of wells designs]. Moscow, Nedra Publ., 1986. 156 p.

7. Magadova L.A. Development of composition for fastening bottomhole zone of layer in wells of underground gas storages. Territoriya NEFTEGAZ, 2012, no. 5, pp. 63-67. In Rus.

8. Yakimov S.B. Sand separators for protection of submersible pumps. Current situation and prospects of use of technology. Territoriya NEFTEGAZ, 2014, no. 2, pp. 44 - 58. In Rus.

9. Atrushkevich S.A. Ejector cleaning wells. Experience of development and application. Vremya koltyubinga. Vremya GRP, 2017, no. 3 (061), pp. 26-27. In Rus.
10. ACTive - Semeystvo aktivnykh servisov GNKT [ACTive is the family of CT active services]. Schlumberger. 2019. Available at: https://www.slb.ru/upload/iblock/4b5/active.pdf (accessed 28 May 208).

11. Bohnhorst D. Hydrodynamische Kanalreinigungsgerate [Hydrodynamic sewer cleaning devices]. Stadtehygiene, 1961, no. 11, pp. $233-240$.

12. Ali A., Blount C.G., Hill S., Pokhrival J., Weng X., Loveland M.J., Mokhtar S., Pedota J., Rodsjo M., Rolovic R., Zhou W. Integrated Wellbore Cleanout Systems: Improving Efficiency and Reducing Risk. Oilfield Review, 2005, vol. 17, no. 2, pp. 4-13.

13. Donald W. Pipe cleaning. Pat. 3744723 US on the invention, 1973.

14. Masters Ph.C. Nozzle. Pat. 3814330 US on the invention, 1974.

15. Hammelmann P. Vorrichtung zum Reinigen von Rohren, Kanalen, Behaltern sowie der Siebe und Filze von Papiermaschinen [Containers and sieves and felts of paper machines]. Pat. $400028 \mathrm{CH}$ hauptpatent, 1966.

16. Bulchaev N.D. Zashchita nasosnogo oborudovaniya neftyanykh skvazhin v oslozhnennykh usloviyakh ekspluatatsii [Protection of pumping equipment of oil wells in complicated operating conditions]. Krasnoyarsk, SFU Publ., 2015. 138 p.

17. Valeev M.D., Vedernikov V.Y., Bulchaev N.D., Salimgareev S.M., Gaskarov V.Z. Klapan obratnoy elektrotsentrobezhnoy ustanovki $i$ sposob ochistki filtra na prieme nasosa [The reverse valve of the electric centrifugal installation and the way of cleaning the filter at the pump intake]. Patent RF, no. 2544930, 2015. 
18. Azeev A.A. Automation of process of cleaning of the intra borehole equipment on the basis of the valve with the analog mechanism. Gazovaya promyshlennost, 2016, no. 4, pp. 82-87. In Rus.

19. Penberthy W.L., Shaughnessy C.M. Sand Control. S P E Series on Special Topics. Society of Petroleum Engineers Publ., 1992. Vol. $1,98 \mathrm{p}$.

20. Acock A., O'rourke T., Shirmboh D. Practical Approaches to Sand Management. Oilfield Review, 2004, vol. 16, no. 1, pp. 10-27.

21. Harrisberger C. Jr, Harrisberger W. Stability of sand arches: a key to sand control. Journal of Petroleum Technology, 1970, vol. 22 (7), pp. 821-829.

22. Smolnikov S.V. Povyshenie effektivnosti ekspluatatsii productivnykh plastov slozhennykh slabostsementirovannymi peschannika$m i$. Avtoreferat Dis. Kand. nauk [Increase in efficiency of operation of the productive layers put by slightly cemented sandstones. Cand. Diss. Abstract]. Moscow, 2015. 24 p.

\section{Information about the authors}

Alexander A. Azeev, Cand. Sc., associate professor, Siberian Federal University.

Nurdi J. Bulchaev, Cand. Sc., head of department, Siberian Federal University.

Elena $\boldsymbol{V}$. Bezverkhaya, Cand. Sc., associate professor, Siberian Federal University.
23. Litvinenko K.B. Prognozirovanie tekhnicheskogo sostoyania UECN $v$ usloviyakh intensivnogo vynosa mekhprimesey. Avtoreferat Dis. Kand. nauk [Forecasting of technical condition of ESP in the conditions of intensive carrying out of mechanical impurity. Cand. Diss. Abstract]. Ufa, 2016. 24 p.

24. Azeev A.A. Povyshenie proizvoditelnosti kompleksa oborudovaniya dlya bestransheynogo remonta truboprovodov sposobom kombinirovannogo toroobraznogo rukava. Avtoreferat Dis. Kand. nauk [Increasing the productivity of a complex of equipment for trenchless pipeline repair using the method of a combined toroidal liner. Cand. Diss. Abstract]. Bratsk, 2011. 23 p.

Received: 29 May 2018. 